\title{
In vivo depletion of CD25+ cells prior to LRAST increases therapeutic efficacy in a murine melanoma model
}

\author{
Julia R Kovács ${ }^{1 *}$, Peter Rose ${ }^{1}$, Louis Boon², Rudolf A Hatz ${ }^{1}$, Hauke Winter ${ }^{1}$, Natasja K van den Engel ${ }^{1}$ \\ From Society for Immunotherapy of Cancer 29th Annual Meeting \\ National Harbor, MD, USA. 6-9 November 2014
}

Lymphodepletion, immune reconstitution and activespecific tumor cell vaccination (LRAST) have proven to benefit the activation of tumor-specific $\mathrm{T}$ cells in mice during homeostatic proliferation. Immunoregulatory mechanisms like the induction of regulatory $\mathrm{T}$ cells may counteract this beneficial effect. To further clarify the role of regulatory $\mathrm{T}$ cells (Treg) during LRAST, we inhibited $\mathrm{CD} 25^{+}$cells in donor mice and/or in recipient mice in vivo, using the monoclonal anti-CD25 antibody PC61. We investigated the induction of an anti-tumor immune response by analyzing tumor growth and the induction of tumor-specific T cells following LRAST.

C57BL/6 recipient mice were pre-treated with a mono-

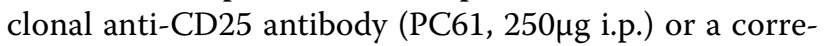
sponding isotype control (anti- $\beta \mathrm{Gal}, 260 \mu$ g, i.p.) twice before tumor inoculation with murine melanoma cells (D5, $5 \times 10^{4}$ cells s.c.). Three days later, lymphopenia was induced by injection of cyclophosphamide $(200 \mathrm{mg} / \mathrm{kg})$. The next day, mice were reconstituted with spleen cells from a congenic mouse strain (C57BL6-Ly5.1, 2x10 ${ }^{7}$ cells i.v.) either pre-treated with anti-CD25 or the isotype antibody. The effect of the anti-CD25 antibody on CD45 ${ }^{+} \mathrm{CD} 4$ ${ }^{+} \mathrm{CD} 25^{+} \mathrm{Foxp}^{+}$(Treg) levels in the peripheral blood was analyzed by flow cytometry. Tumor growth was monitored and the induction of tumor-specific $\mathrm{T}$ cells was analyzed by IFN- $\gamma$ cytokine release assay.

Three of five $(60 \%)$ of the mice treated with anti-CD25 antibody and reconstituted with spleen cells from Tregdepleted donor mice showed a prolonged survival in comparison to mice treated with isotype antibody and reconstituted with spleen cells from naïve donors. Tregdepleted hosts reconstituted with cells from donor mice treated with an isotype control showed the highest release of tumor-specific IFN- $\gamma$ in tumor-draining lymph node cells. Interestingly, reconstitution with anti-CD25depleted spleen cells significantly reduced tumor-specific IFN- $\gamma$ release in tumor-draining lymph node cells.

Treg inactivation with anti-CD25 antibody prior to LRAST increases therapeutic efficacy. These results suggest that CD25 depletion of the host, but not of the donor, is advantageous for the induction of a tumor-specific immune response. Whether this treatment protocol results in a therapeutic immune response and further improves survival is currently under investigation.

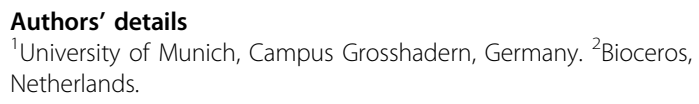

Published: 6 November 2014

\section{doi:10.1186/2051-1426-2-S3-P51}

Cite this article as: Kovács et al:: In vivo depletion of CD25+ cells prior to LRAST increases therapeutic efficacy in a murine melanoma model. Journal for ImmunoTherapy of Cancer 2014 2(Suppl 3):P51.

${ }^{1}$ University of Munich, Campus Grosshadern, Germany

Full list of author information is available at the end of the article 